\title{
Boosting the Searches of Gluon Pair Initiated Processes using Deep Learning of ISR Jets
}

\section{Dongsub Lee*, Won Sang Cho, Hyung Do Kim}

Dept. Physics and Astronomy, Seoul National University

E-mail: dongsub93esnu.ac.kr

\begin{abstract}
We introduce new strategy to improve the searches of the signal from gluon-pair-initiated(ggI) processes at the LHC. By the ggI process, we mean the scattering process starting from a pair of gluons which can be associated with initial-state radiation jets(ISR jets) emitted from various incident parton at hadron collision. The ggI processes include gluon-gluon fusion( $\mathrm{ggF}$ ) into colour singlet state, Higgs associated top-quark pair productions, which are the important windows of proving the sector of electroweak symmetry breaking. Focusing on the flavours of associated to each ISR jets, we show that the ISR jets in the ggI processes have distinctive features from background processes. Provided that features, we classified initial states, and discriminated the ggI processes from its backgrounds by using deep neural networks. As a promising example, we demonstrated this new technique on single Higgs production process in dimuon channel. Higgs to dimuon process is elusive because of huge irreducible backgrounds from Drell-Yan and diboson processes. Finally, we provide an enhanced aspect on the Higgs coupling measurements, and discuss on the universality of this technique for probing ggI processes in various channels.
\end{abstract}

The 39th International Conference on High Energy Physics (ICHEP2018)

4-11 July, 2018

Seoul, Korea

${ }^{*}$ Speaker. 


\section{Introduction}

At the Large Hadron Collider(LHC) process with different initiating particles have jet started from different particles, and this can enhance signal extraction efficiency. We demonstrate this technique on simplified Higgs-to-dimuon analysis with Drell-Yan background and signal from Higgs produced by gluon-gluon fusion.

\section{Jet Flavour Tagging}

Based on the works on, for examples, [1] and [2], there are variables related to substructure variables which are known to have discrimination power of initial particles of jets. But they do not have clear separation, we need to do multi-variate analysis(MVA) with them on top of the other variables. As an algorithm for MVA, we choose deep neural network(DNN).

\section{Event Generation and Selection}

Both are generated by MadGraph5 aMC@NLO[3] at fully next-to-leading(NLO) order and merging is done with FxFx scheme[4]. For signal Higgs Chraterisation[5] was used and showered with Pythia8[6] and detector simulation is done by Delphes[7]. Pre-selection criteria and signal window is chosen following one used in [8].

\section{Result and Conclusion}

We checked almost $10 \%$ enhancement of $S / \sqrt{B}$ with derived background rejection rate as a function of signal efficiency from prediction model and number of events normalised to $35.9 \mathrm{fb}^{-1}$ with $\sqrt{s}=14 \mathrm{TeV}$ from most of signal efficiency region. From this we can conclude the original motivation is true and it does help the analysis.

\section{References}

[1] Jason Gallicchio and Matthew D. Schwartz, Phys. Rev. Lett. 107172001 (2011)

[2] Biplob Bhattacherjee, Satyanarayan Mukhopadhyay, Mihoko M. Nojiri, Yasuhito Sakaki, Bryan R. Webber, JHEP 1504 (2015) 131

[3] J. Alwall, R. Frederix, S. Frixione, V. Hirschi, F. Maltoni, O. Mattelaer, H.-S. Shao, T. Stelzer, P. Torrielli, M. Zaro, arXiv:hep-ph/1405.0301

[4] Rikkert Frederix, Stefano Frixione, arVix:hep-ph/1209.6215

[5] F. Demartin, B. Maier, F. Maltoni, K. Mawatari, M. Zaro, Eur. Phys. J 77 (2017) 34.

[6] T. Sjöstrand, S. Mrenna and P. Skands, JHEP05 (2006) 026, Comput. Phys. Comm. 178 (2008) 852.

[7] J. de Favereau, C. Delaere, P. Demin, A. Giammanco, V. LemaÃôtre, A. Mertens, M. Selvaggi, arXiv:hep-ex/1307.6346

[8] CMS Collaboration, CMS-PAS-HIG-17-019(2017) 